\title{
Impact of conscious sedation and general anesthesia on periprocedural outcomes in Watchman left atrial appendage closure
}

\author{
Caroline Kleinecke ${ }^{1 *}$, Wasim Allakkis ${ }^{2 *}$, Eric Buffle $^{3}, \mathrm{Xiao}_{\mathrm{Xia}} \mathrm{Liu}^{2,4}$, \\ Yamen Mohrez ${ }^{2}$, Steffen Gloekler ${ }^{3}$, Johannes Brachmann ${ }^{2}$, \\ Steffen Schnupp ${ }^{2}$, Stephan Achenbach ${ }^{5}$, Jiangtao $\mathrm{Yu}^{1,6}$ \\ ${ }^{1}$ Department of Cardiology, REGIOMED Klinikum Lichtenfels, Lichtenfels, Germany \\ ${ }^{2}$ Department of Cardiology, REGIOMED Klinikum Coburg, Coburg, Germany \\ ${ }^{3}$ Department of Cardiology, University Hospital of Bern, Bern, Switzerland \\ ${ }^{4}$ Department of Cardiology, Beijing Anzhen Hospital, Beijing, China \\ ${ }^{5}$ Department of Cardiology, Friedrich-Alexander University Erlangen-Nuernberg, Erlangen, Germany \\ ${ }^{6}$ Department of Cardiology, Klinikum Koblenz-Montabaur, Koblenz, Germany
}

\begin{abstract}
Background: Transcatheter left atrial appendage closure (LAAC) is performed either in conscious sedation (CS) or general anesthesia (GA), and limited data exist regarding clinical outcomes for the two approaches. The aim of the study was to analyze the effect of CS versus GA on acute outcomes in a large patient cohort undergoing LAAC with a Watchman occluder.

Methods: A cohort of 521 consecutive patients underwent LAAC with Watchman occluders at two centers (REGIOMED hospitals, Germany) between 2012 and 2018. One site performed 303 consecutive LAAC procedures in GA, and the other site performed 218 consecutive procedures in CS. The safety endpoint was a composite of major periprocedural complications and postoperative pneumonia. The efficacy endpoint was defined as device success.

Results: After a 1:1 propensity score matching, 196 (CS) vs. 115 (GA) patients could be compared. In $5(2.6 \%)$ cases CS was converted to GA. The primary safety endpoint (3.5\% [CS] vs. $7.0 \%$ [GA], $p=0.18$ ) and its components (major periprocedural complications: $2.5 \%$ vs. $3.5 \%, p=0.73$; postoperative pneumonia: $2.6 \%$ vs. $4.3 \%, p=0.51$ ) did not differ between the groups. Also, device success was comparable (96.9\% vs. $93.9 \%, p=0.24)$.

Conclusions: In patients undergoing LAAC with the Watchman device, conscious sedation and general anesthesia showed comparable device success rates and safety outcomes. The type of anesthesia for LAAC may therefore be tailored to patient comorbidities, operator experience, and hospital logistics. (Cardiol J 2021; 28, 4: 519-527)
\end{abstract}

Key words: atrial fibrillation, left atrial appendage closure, conscious sedation, general anesthesia, Watchman

\footnotetext{
Address for correspondence: Caroline Kleinecke, MD, Department of Cardiology, Klinikum Lichtenfels, Professor-Arneth-Straße 2b, 962215, Lichtenfels, Germany, tel: +49 957112 9419, fax: +49 957112 2909, e-mail: carolinekleinecke@web.de Received: 8.11.2020 Accepted: 3.12.2020

*The first two authors contributed equally to this work.

This article is available in open access under Creative Common Attribution-Non-Commercial-No Derivatives 4.0 International (CC BY-NC-ND 4.0) license, allowing to download articles and share them with others as long as they credit the authors and the publisher, but without permission to change them in any way or use them commercially.
} 


\section{Introduction}

Left atrial appendage closure (LAAC) is a device-based method for stroke prevention in patients with atrial fibrillation (AF), who have absolute or relative contraindications for oral anticoagulation (OAC) [1, 2]. Substantial evidence exists for the Watchman occluder (Boston Scientific, Marlborough, MA, US), which has been in clinical use since 2005. Compared to warfarin, it provides comparable efficacy in long-term prevention of all-cause stroke. Furthermore, it reduces cardiovascular mortality and major bleeding events, particularly hemorrhagic stroke [3]. Because LAAC is a purely preventive treatment, without any immediate benefit for the patient, it is mandatory to keep periprocedural complications as low as possible. Due to the complex anatomy and topography of the left atrial appendage (LAA), the procedure can be demanding. Procedural success and adverse events depend on several factors, such as patient characteristics and comorbidities, experience of the operating physician and team, technical features of the device, intraprocedural cardiac imaging, and possibly the type of anesthesia. Most centers prefer procedural guidance by transesophageal echocardiography (TEE), which requires either sedation or full anesthesia to improve patient comfort and tolerance of the TEE probe. One option is general anesthesia (GA), which allows control of airways, ventilation, and patient movement. The other is conscious sedation (CS) with the patient spontaneously breathing. Previous studies investigating the type of anesthesia used during other heart disease interventions could not establish superiority of any one method but documented shorter postoperative monitoring or length of hospital stay in CS [4-10]. Data on the impact of CS or GA on periprocedural outcomes in LAAC are lacking. Therefore, the aim of this study was to compare both methods with regard to device success and major periprocedural complications, including postoperative pneumonia, as well as length of postoperative monitoring based on the results of two propensity-matched, real-world registries.

\section{Methods}

\section{Study cohort}

All consecutive patients, who underwent LAAC with Watchman occluders at two centers (REGIOMED hospitals Coburg and Lichtenfels, Germany), were prospectively enrolled in an observational registry. At the Lichtenfels site, where inclusion started in 2012, all patients were treated exclusively under GA. The Watchman program at the Coburg site started in 2016, and all interventions were performed primarily under conscious sedation (Fig. 1). Indications for LAAC were based on current guidelines and recommendations [1,2]. Exclusion criteria were active infection, pregnancy, and indications for OAC other than AF. A retrospective post-hoc analysis of demographic characteristics, procedural data, and clinical outcomes was performed for all patients in a standardized manner. Adverse events were adjudicated by a clinical event committee of two independent physicians and, in cases of disagreement, by a third referee. The study complies with the Declaration of Helsinki. It was conducted according to local ethical standards and requirements, and all patients provided written informed consent.

\section{LAAC procedure}

The characteristics of the Watchman device and procedural aspects were previously described in detail [11]. Generally, the occluder was implanted via a transseptal puncture and use of a delivery sheath. In both groups, all procedures were performed in a catheterization laboratory and were both guided by TEE and fluoroscopy. However, procedural settings, postoperative monitoring, and antithrombotic medical therapy differed between the two centers: For the GA group, the team comprised 3 to 4 doctors ( 1 or 2 interventional cardiologists, one TEE guide, one anesthesiologist) and 4 nurses (Fig. 2). All patients were orotracheally intubated and mechanically ventilated. In the majority of cases, the anesthesia was a combination of propofol and an opioid (fentanyl or remifentanil). Extubation was aimed to be performed in the catheterization laboratory immediately after the procedure, whenever possible. The standard protocol provided a postoperative monitoring in the intermediate care (IMC) unit for 24 hours. According to the PROTECT-AF study, postoperative antithrombotic therapy consisted of OAC plus acetylsalicylic acid (ASA) for 45 days followed by dual antiplatelet therapy with ASA and clopidogrel for 6 months [12]. In the CS group, the operating team consisted of 3 cardiologists ( 2 interventionalists, 1 TEE guide) and 3 nurses, all with expertise in propofol sedation and intensive care unit (ICU) skills (Fig. 2). All patients received local anesthesia with mepivacaine $1 \%$ at the puncture site and lidocaine pump spray for introduction of the TEE probe. Patients were spontaneously breathing, and conversion to 


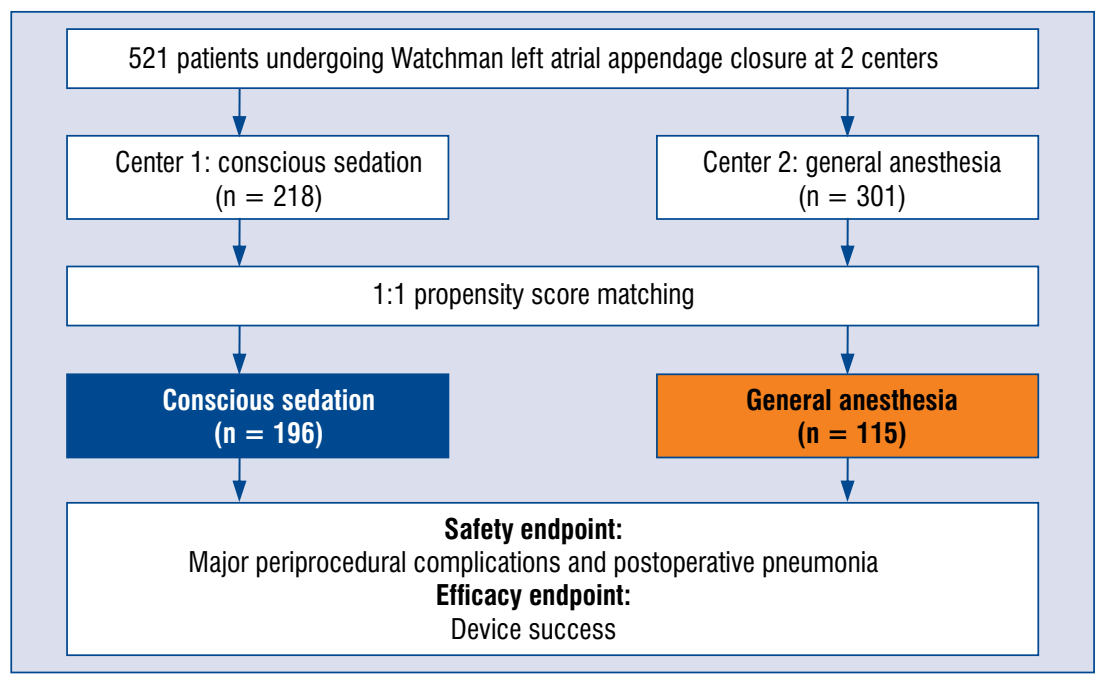

Figure 1. Study flow chart.

GA was only performed in case of respiratory or hemodynamic instability. For sedation, a $1-5 \mathrm{mg}$ bolus of midazolam was administered. In addition, propofol was administered continuously at the lowest dose possible. The standard protocol required 4 hours of postoperative monitoring in the IMC unit. The postprocedural antithrombotic regimen consisted of dual antiplatelet therapy with ASA and clopidogrel for 3 months.

\section{Definitions and endpoints}

Demographic, clinical, and procedural characteristics, as well as adverse events and endpoints, were reported according to the current recommendations of the European Heart Rhythm Association and the European Association of Percutaneous Cardiovascular Interventions, the Bleeding Academic Research Consortium (BARC), the Valve Academic Research Consortium criteria, and the Cardiovascular and Stroke Endpoint Definitions for Clinical Trials [13-16]. Device success was defined as correct deployment and implantation of the LAA occluder. Major periprocedural complications included death $(<72$ hours after the index procedure), stroke, device embolization, cardiac tamponade or pericardial effusion requiring intervention, major bleeding (> BARC type 3a), need for bailout surgery, need for cardio-pulmonary resuscitation, severe kidney injury, and other relevant complications leading to prolonged hospital stay. The safety endpoint was a composite of the mentioned major periprocedural complications and postoperative pneumonia. The efficacy endpoint was defined as device success.

\section{Statistical analysis}

Statistical analyses were performed with the Graph Pad Prism 8 software (GraphPad Inc. La Jolla, California, USA). Categorical variables are presented as actual numbers and percentages and compared using the Fisher's exact test. Continuous variables are summarized as mean \pm standard deviation (SD) or as medians with corresponding interquartile range (IQR) and compared using an unpaired t-test. Findings were considered statistically significant at the 0.05 level. We performed a propensity score matching for the likelihood of performing LAAC under conscious sedation or general anesthesia with a ratio of $1: 1$ with replacement using the R software, the package "MatchIt" and "cobalt" [17-19]. The quality of the matching was assessed in two ways: there was a standardized difference of $<0.10$ for each baseline covariable and there was no significant difference in the covariables among the two groups using a univariate logistic regression or unpaired t-test.

\section{Results}

\section{Patient characteristics}

Between November 2016 and May 2018, 218 patients underwent LAAC with Watchman occluders under CS at the Coburg site, and respectively 303 received Watchman devices under GA at the Lichtenfels site, between February 2012 and April 2017. After a 1:1 propensity score matching, 196 (CS) vs. 115 (GA) patients were compared (Fig. 1). Baseline characteristics are shown in Table 1 . The prevalence of female gender $(49.0 \%$ [CS] vs. $36.5 \%$ 
Table 1. Baseline characteristics.

\begin{tabular}{lccc}
\hline & $\begin{array}{c}\text { Conscious sedation } \\
(\mathbf{n}=196)\end{array}$ & $\begin{array}{c}\text { General anesthesia } \\
\text { (n = 115) }\end{array}$ & P \\
\hline Age at time of LAAC [years] & $78.0 \pm 7.3$ & $77.0 \pm 6.8$ & 0.23 \\
Female gender & $96(49.0 \%)$ & $42(36.5 \%)$ & 0.03 \\
Body mass index $\left[\mathrm{kg} / \mathrm{m}^{2}\right]$ & $29.7 \pm 6.4$ & $29.5 \pm 5.1$ & 0.69 \\
Permanent/persistent AF & $105(53.6 \%)$ & $77(67.0 \%)$ & 0.02 \\
Arterial hypertension & $185(94.4 \%)$ & $101(87.8 \%)$ & 0.04 \\
Diabetes mellitus & $67(34.2 \%)$ & $40(34.8 \%)$ & 0.91 \\
Coronary artery disease & $113(57.7 \%)$ & $60(52.2 \%)$ & 0.35 \\
Prior PCl/CAGB & $88(44.9 \%)$ & $47(40.9 \%)$ & 0.49 \\
LVEF [\%] & $53.1 \pm 11.9$ & $55.1 \pm 9.5$ & 0.12 \\
Congestive heart failure & $59(30.1 \%)$ & $29(25.2 \%)$ & 0.36 \\
Glomerular filtration rate $[\mathrm{mL} / \mathrm{min}]$ & $55.3 \pm 26.0$ & $56.2 \pm 22.8$ & 0.75 \\
Severe lung disease & $14(7.1 \%)$ & $11(9.6 \%)$ & 0.45 \\
Liver disease & $12(6.1 \%)$ & $17(14.8 \%)$ & 0.01 \\
CHA ${ }_{2}$ DS ${ }_{2}$-VASc score & $4.8 \pm 1.4$ & $4.6 \pm 1.7$ & 0.40 \\
HAS-BLED score & $3.4 \pm 0.8$ & $3.4 \pm 0.9$ & 0.63 \\
\hline
\end{tabular}

Categorical variables are expressed as frequencies $(n)$ and percentages (\%). Continuous data is reported as mean and standard deviation; $\mathrm{AF}$ - atrial fibrillation; CABG — coronary artery bypass grafting; LAAC - left atrial appendage closure; LVEF — left ventricular ejection fraction; $\mathrm{PCl}$ - percutaneous coronary intervention

[GA], $\mathrm{p}=0.03)$ and arterial hypertension $(94.4 \%$ vs. $87.8 \%, p=0.04$ ) was higher in the CS group. The rate of permanent/persistent $\mathrm{AF}$ (53.6\% vs. $67.0 \%, \mathrm{p}=0.02)$ and liver disease $(6.1 \%$ vs. $14.8 \%$, $\mathrm{p}=0.01$ ) was higher in the GA group. All other baseline characteristics were comparable, especially age $(78.0 \pm 7.3[\mathrm{CS}]$ vs. $77.0 \pm 6.8[\mathrm{GA}], \mathrm{p}=$ $=0.23)$, stroke and bleeding risk $\left(\mathrm{CHA}_{2} \mathrm{DS}_{2}\right.$-VASC score: $4.8 \pm 1.4$ vs. $4.6 \pm 1.7, \mathrm{p}=0.40$; HAS-BLED score: $3.4 \pm 0.8$ vs. $3.4 \pm 0.9, \mathrm{p}=0.63)$, as well as renal function (glomerular filtration rate $[\mathrm{mL} / \mathrm{min}]$ : $55.3 \pm 26.0$ vs. $56.2 \pm 22.8, \mathrm{p}=0.75)$ and severe lung disease $(7.1 \%$ vs. $9.6 \%, \mathrm{p}=0.52)$.

\section{Procedural characteristics}

Procedural aspects are depicted in Table 2. All interventions were guided by TEE, apart from 1 case of intracardiac echocardiography in the CS group. In the CS group the rate of implantation attempts $(1.2 \pm 0.6[\mathrm{CS}]$ vs. $1.6 \pm 0.9[\mathrm{GA}], \mathrm{p} \leq$ $\leq 0.001)$ and use of contrast media were lower (median, mL: 50 [IQR 30-60] vs. 90 [IQR 70-113], $\mathrm{p} \leq 0.001$ ); furthermore, fluoroscopy (median, min: 7 [IQR 5-10] vs. 10 [IQR 7-19], $\mathrm{p} \leq 0.001)$ and procedure (median, min: 41 [IQR $35-55]$ vs. 49 [IQR 38-65], $\mathrm{p}=0.02$ ) times were shorter. In $5(2.6 \%)$ patients CS was converted to GA: in 1 case due to LAA perforation with cardiogenic shock, in another due to epistaxis with aspira- tion, and in 3 patients due to respiratory failure. The primary safety endpoint $(3.5 \%$ [CS] vs. $7.0 \%$ [GA], $p=0.18$ ) did not differ significantly between the groups. Also, its components, postoperative pneumonia ( $2.6 \%$ vs. $4.3 \%, \mathrm{p}=0.51$ ), and major periprocedural complications $(2.5 \%$ vs. $3.5 \%$, $\mathrm{p}=0.73$ ) were comparable (Fig. 3 ).

In the CS group, 1 case of procedure-related death occurred in an 87-year-old, female patient, who suffered from a major stroke shortly after the intervention. Cerebral computed tomography after 24 hours demonstrated relevant ischemic cerebral infarction, and the patient died 3 days after the index procedure. Device success was comparable between the groups $(96.9 \%$ vs. $93.9 \%, \mathrm{p}=0.24)$. As provided by the standard protocol of each center, postoperative monitoring was significantly shorter in the CS group (median, hours: $4.0 \pm 59.9$ vs. $24.0 \pm 10.8, p=0.03$; Fig. 3 ). Both groups show large standard deviations due to patients with periprocedural complications, who were treated for several days or weeks in the ICU.

\section{TEE follow-up and 30-day mortality}

Due to logistic reasons, the TEE follow-up rate is incomplete in the CS group and differs between the groups $(62.2 \%$ [CS] vs. $100 \%$ [GA], $\mathrm{p} \leq 0.0001)$ (Table 3$)$. Therefore, a numerically lower rate of device-related thrombus was observed in the CS 
Table 2. Procedural characteristics.

\begin{tabular}{|c|c|c|c|}
\hline & $\begin{array}{l}\text { Conscious sedation } \\
\text { (n = 196) }\end{array}$ & $\begin{array}{l}\text { General anesthesia } \\
\text { ( } \mathrm{n}=115)\end{array}$ & $\mathbf{P}$ \\
\hline Conversion from CS to GA & $5(2.6 \%)$ & & \\
\hline TEE guidance & $194(99.5 \%)$ & $115(100.0 \%)$ & 0.44 \\
\hline Device success & $190(96.9 \%)$ & $108(93.9 \%)$ & 0.20 \\
\hline Implantation attempts & $1.2 \pm 0.6$ & $1.6 \pm 0.9$ & $<0.0001$ \\
\hline Fluoroscopy time [min] & 7 (IOR 5-10) & 10 (IQR 7-19) & $<0.0001$ \\
\hline Procedure time [min] & 41 (IOR 35-55) & 49 (IOR 38-65) & 0.0002 \\
\hline Total contrast volume $[\mathrm{mL}]$ & 50 (IOR 30-60) & 90 (IQR 70-113) & $<0.0001$ \\
\hline Postoperative monitoring [h] & 4 (IOR 4-4) & 24 (IOR 22-24) & 0.03 \\
\hline Length of hospital stay [days] & 13 (IQR 6-16) & 3 (IOR 3-8) & $<0.0001$ \\
\hline Primary safety endpoint & $7(3.5 \%)$ & $8(7.0 \%)$ & 0.18 \\
\hline Postoperative pneumonia & $5(2.6 \%)$ & $5(4.3 \%)$ & 0.39 \\
\hline Major periprocedural complication: & $5(2.5 \%)$ & $4(3.5 \%)$ & 0.64 \\
\hline Death & $1(0.5 \%)$ & $0(0.0 \%)$ & 0.44 \\
\hline Stroke & $1(0.5 \%)$ & $0(0.0 \%)$ & 0.44 \\
\hline Pericardial tamponade & $2(1.0 \%)$ & $4(3.5 \%)$ & 0.13 \\
\hline Major bleeding & $3(1.5 \%)$ & $4(3.5 \%)$ & 0.26 \\
\hline Major access vessel complication & $1(0.5 \%)$ & $1(0.9 \%)$ & 0.70 \\
\hline Need for bailout surgery & $0(0.0 \%)$ & $0(0.0 \%)$ & 1.0 \\
\hline Device embolization & $0(0.0 \%)$ & $0(0.0 \%)$ & 1.0 \\
\hline Severe kidney injury & $0(0.0 \%)$ & $1(0.9 \%)$ & 0.44 \\
\hline Cardiogenic shock & $2(1.0 \%)$ & $0(0.0 \%)$ & 0.28 \\
\hline Need for cardiopulmonary resuscitation & $1(2.5 \%)$ & $0(0.0 \%)$ & 0.28 \\
\hline
\end{tabular}

Categorical variables are expressed as frequencies $(\mathrm{n})$ and percentages $(\%)$. Continuous data is reported as mean and standard deviation or as median and interquartile range; CS — conscious sedation; GA — general anesthesia; TEE — transesophageal echocardiography

group $(0.8 \%$ vs. $5.2 \%, \mathrm{p}=0.01)$. The rate of major peri-device leaks $(\geq 5 \mathrm{~mm})$ was comparable between the groups $(2.8 \%$ vs. $1.4 \%, \mathrm{p}=0.47)$. Thirty-day mortality was similar for both groups $(3.6 \%$ vs. $1.4 \%, \mathrm{p}=0.47$ ) (Fig. 4 ): In the CS group, 7 patients had died at 30-day follow-up for the following reasons: unexplained death ( 80 and 88 years old, both male), heart failure ( 85 and 75 years old, both male), liver failure (70 years old, male), pancreatic cancer (86 years old, male), and stroke (87 years old, female). In the GA group, a 71-year-old male patient died due to unexplained reasons, a 70-year-old male patient due to pneumonia 3 weeks after the index procedure, and an 87-year-old female patient due to spontaneous retroperitoneal hematoma 25 days after the index procedure.

\section{Discussion}

Over the last decade, LAAC has become an accepted procedure and is offered in many centers. Commonly, procedural imaging is necessary to guide this often complex intervention. One guiding tool is intracardiac echocardiography, which avoids anesthesia and its associated potential risks. However, most centers use TEE, which is widely available, less expensive, and offers superior imaging quality. In many centers, general anesthesia is used to improve patient comfort and tolerance to the procedure.

Currently, there are limited data regarding the impact of the anesthesiologic strategy in LAAC on procedural, peri-procedural, and later clinical outcomes: A case series in 11 patients investigated LAAC under sedation and noninvasive ventilation [20]. In all patients, clinical outcome was uneventful, with a high degree of satisfaction of the medical team.

The present study is the first that directly compares CS and GA for LAAC with Watchman occluders based on the results of propensity-matched data from two real-world registries.

Our main findings are: (1) CS and GA are equally effective for device success; (2) both meth- 
Cardiology Journal 2021, Vol. 28, No. 4

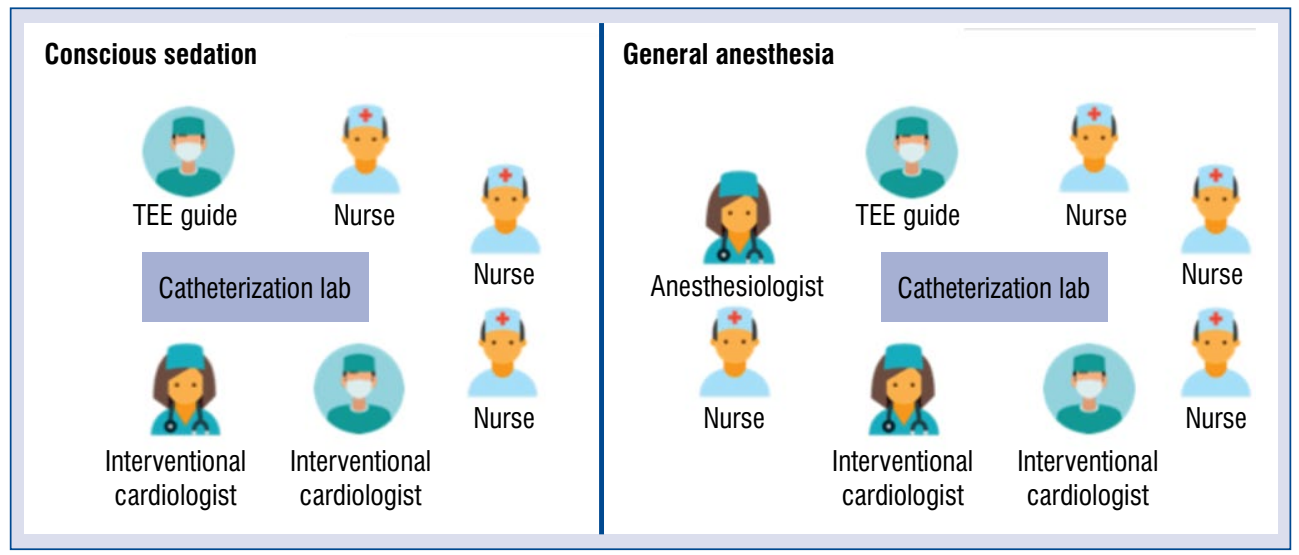

Figure 2. Procedure settings in conscious sedation and general anesthesia; lab — laboratory; TEE — transesophageal echocardiography.

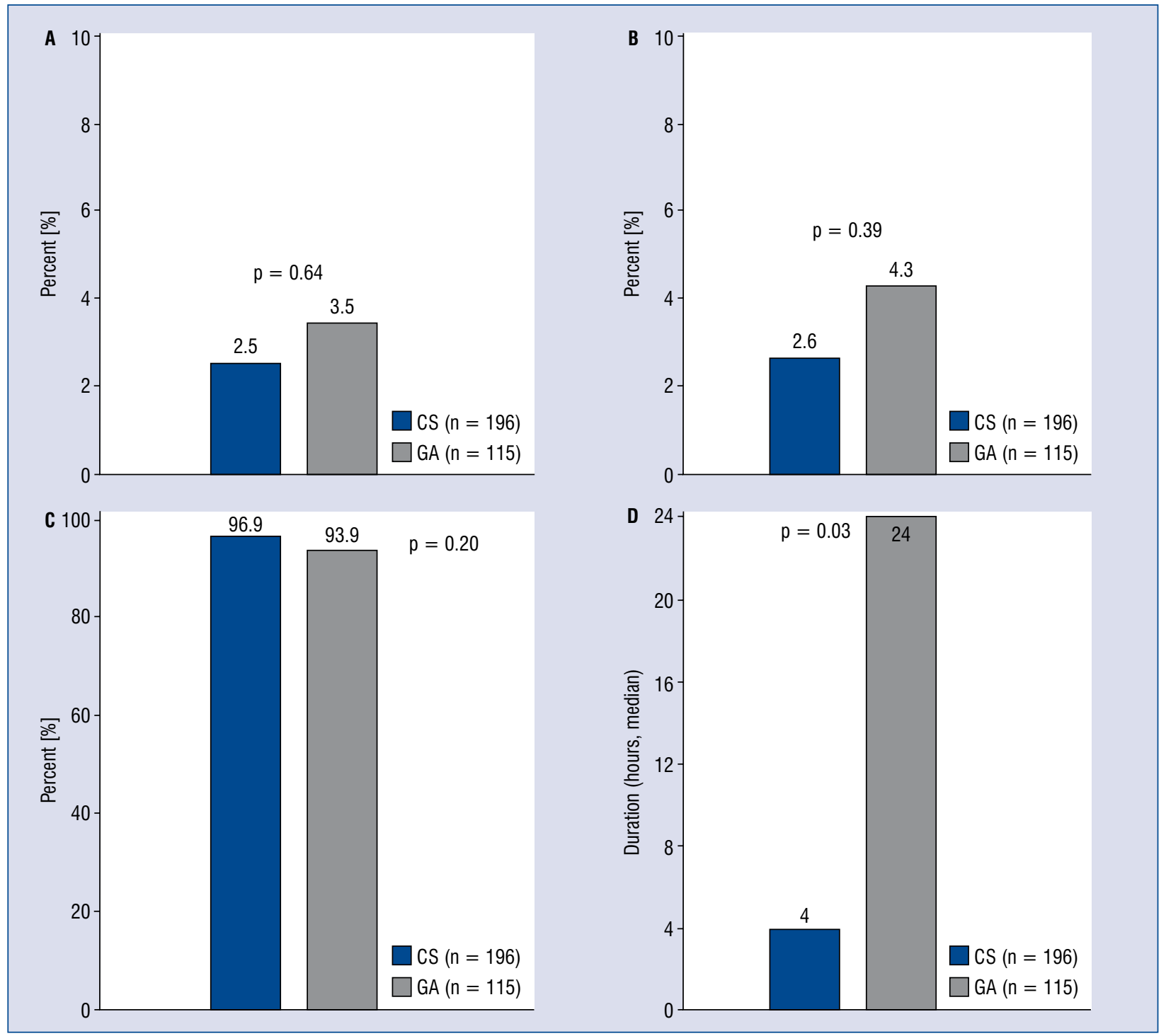

Figure 3. Key variables of periprocedural outcomes; A. Major periprocedural complications; B. Postoperative pneumonia; C. Device success; D. Postoperative monitoring; CS — conscious sedation; GA — general anesthesia. 
Table 3. Transesophageal echocardiography (TEE) follow-up and 30-day mortality.

\begin{tabular}{lccc}
\hline & $\begin{array}{c}\text { Conscious sedation } \\
(\mathbf{n}=196)\end{array}$ & $\begin{array}{c}\text { General anesthesia } \\
\text { (n = 115) }\end{array}$ & P \\
\hline TEE performed & $122(62.2 \%)$ & $115(100.0 \%)$ & $<0.0001$ \\
Device-related thrombus & $1(0.8 \%)$ & $6(5.2 \%)$ & 0.01 \\
Peri-device leak $(\geq 5 \mathrm{~mm})$ & $9(8.6 \%)$ & $9(7.8 \%)$ & 0.24 \\
30-day mortality & $7(3.6 \%)$ & $3(1.4 \%)$ & 0.47 \\
\hline
\end{tabular}

Categorical variables are expressed as frequencies $(n)$ and percentages $(\%)$.

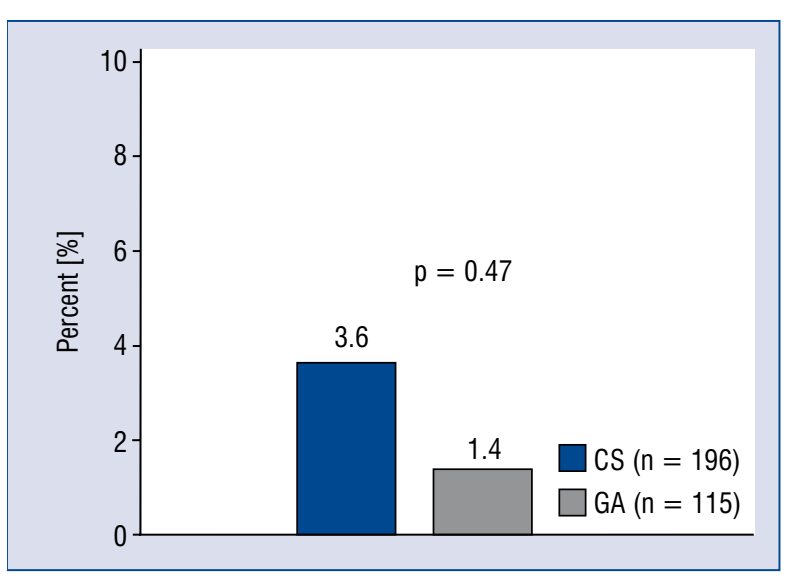

Figure 4. 30-day mortality; CS - conscious sedation; GA - general anesthesia.

ods offer comparable safety with regard to major periprocedural complications and postoperative pneumonia; (3) the type of anesthesia does not affect 30-day mortality; and (4) CS requires shorter postoperative monitoring and less personal and logistic resources.

These observations are in line with other studies that investigated the impact of CS and GA on outcomes in other structural interventions such as percutaneous mitral valve repair or transcatheter aortic valve implantation (TAVI) [4-10]. Those studies report no differences in procedural success or clinical outcomes according to the type of anesthesia. In the present study, device success was comparable to the implant success rate $(95.8 \%)$ of the worldwide largest National Cardiovascular Data Registry (NCDR) with 38,158 procedures, captured between 2016 and 2018 [21]. The NCDR reports a fairly low rate of major periprocedural complications (1.9\%). The rates of adverse events in our cohorts are higher, which may be attributable to the older and more polymorbid patient population of our study with higher $\mathrm{CHA}_{2} \mathrm{DS}_{2}$-VASc and HAS-
-BLED scores and the much shorter timeframe of treatment starting from 2012.

Similar to our results, the German Aortic Valve Registry reported shorter procedure and fluoroscopy times for CS [9]. The United States post-Food and Drug Administration approval Watchman registry of 3822 patients reported a median procedure time of $50 \mathrm{~min}$ [22]. In comparison, our results with a median of $49 \mathrm{~min}$ for the GA and $41 \mathrm{~min}$ for the CS group are comparable. Despite speedier interventions in our CS group arm, we observed fewer implantation attempts and lower contrast volume in this group. The most likely reason for this is that in the GA group patient enrollment started 4 years earlier, which affects expertise with the procedure and explains the differences in operation times, contrast use, and implantation attempts between our two groups. The PROTECT-AF trial and the continued access registry showed - as in all interventional procedures - a learning curve of the performing teams with a substantial improvement in procedural speed and success, as well as a significant decrease in procedure-related complications [23]. However, another potential reason for these findings may be the type of anesthesia: General anesthesia avoids excess salivary secretion and consequent coughing and unexpected moving. This enables a more meticulous transseptal puncture, several measurements of the LAA, as well as repetitive re-positioning or replacement of the device if necessary. Therefore, GA is often deemed more appropriate for operators and hospitals at the beginning of their learning curves, whereas CS, which considerably streamlines the procedure, is a valuable option for high-volume centers with experienced operators and teams.

As outlined, in our CS group, 5 (2.6\%) patients had to be converted to GA. Respiratory failure with the need for temporary non-invasive ventilation or orotracheal intubation is a common adverse effect of deep conscious sedation, but most of these cases recover rapidly and uneventfully. Indeed, all 
3 patients who suffered respiratory depression could be extubated on the same day. Despite choosing $\mathrm{CS}$ as the primary anesthesiologic approach, the team has to be prepared for speedy conversion to GA if necessary. In a single logistic regression analysis, we were unable to detect any predictors for respiratory depression in our group, which is due to the low numbers.

Conceptually, CS might be associated with an increased aspiration risk caused by hypersalivation due to the TEE probe. However, studies investigating GA and CS in patients undergoing percutaneous mitral valve repair observed no differences regarding the development of postoperative pneumonia, which is similar to our findings $[4,6]$. In those studies, the rate of postoperative pneumonia varied between $3.3 \%$ and $6.7 \%$, which is comparable to the rate observed in the present study, in which all patients received peri-interventional prophylactic antibiotics according to the guidelines. Also, for postoperative pneumonia and due to the low event rates, we found no relevant predictors in the logistic regression analysis.

With regard to 30-day mortality, rates were comparable between the groups but higher than observed in the EWOLUTION registry $(0.4 \%)$ [24]. Nonetheless, after 1 year, the EWOLUTION registry reported a relatively high mortality rate of $9.8 \%$, reflecting the elderly, fragile, and multimorbid LAAC patient population in European countries [25]. Individual patient characteristics such as biological and social status, the burden of comorbidities, quality of life, frailty, and anticipated residual lifespan should be considered when planning LAAC in elderly patients. With regard to postoperative monitoring, the standard protocol of the two centers required 4 hours for CS and 24 hours for GA. Other investigations documented consistently shorter IMC/ICU hours after CS only [4-10]. In addition, Toppen et al. [7] reported a higher quality of life at 30-day follow-up and a decrease in direct costs in patients, who received TAVI under CS. However, shorter postoperative monitoring seems not to affect overall length of hospital stay [4]. In the present study, in the GA group LAAC was performed as an elective procedure, whereas in the CS group patients were mostly hospitalized for other reasons, which is clearly the reason for the longer hospital stay in the latter group.

\section{Limitations of the study}

Due to its retrospective, non-randomized, and observational design, as well as the different proce- dural settings of the GA and CS groups, the present study has several limitations. Due to its relatively small sample size, our study was not powered to detect differences in procedural and clinical outcomes. Despite good comparability of the two groups with regard to baseline characteristic due to propensity score matching, confounders may persist, e.g. differences in the rates of gender, arterial hypertension, permanent $\mathrm{AF}$, and liver disease. Furthermore and importantly, at the Lichtenfels site, patient enrollment started 4 years earlier and included the learning curve of the center, which likely impacted on the longer intervention and fluoroscopy times, as well as more implantation attempts and the use of greater amounts of contrast volume in this group. Regarding TEE follow-up, it was incomplete in the CS group and was not analyzed in a core laboratory, which may have led to an underreporting of device-related thrombus and peri-device leaks in this group.

\section{Conclusions}

In patients undergoing LAAC with the Watchman occluder, general anesthesia and conscious sedation provided comparable safety, efficacy, and device success, but CS shortened postoperative monitoring. Therefore, the type of anesthesia for LAAC may be tailored to patient characteristics, operator experience, and hospital logistics.

Conflict of interest: Steffen Gloekler has received a grant from the Swiss Heart Foundation; Johannes Brachmann has received consulting fees from Abbott, St.Jude Medical, Medtronic, Bayer, Liva-nova, Pfizer, Boston Scientific, Boehringer Ingelheim, and Biotronik; Jiangtao Yu has received proctor fees from Boston Scientific. The other authors have no conflicts of interest.

\section{References}

1. Kirchhof P, Benussi S, Kotecha D, et al. ESC Scientific Document Group. 2016 ESC Guidelines for the management of atrial fibrillation developed in collaboration with EACTS. Eur Heart J. 2016; 37(38): 2893-2962, doi: 10.1093/eurheartj/ehw210, indexed in Pubmed: 27567408.

2. Glikson M, Wolff R, Hindricks G, et al. EHRA/EAPCI expert consensus statement on catheter-based left atrial appendage occlusion - an update. EuroIntervention. 2020; 15(13): 1133-1180, doi: 10.4244/EIJY19M08_01, indexed in Pubmed: 31474583.

3. Reddy VY, Doshi SK, Kar S, et al. 5-Year outcomes after left atrial appendage closure: from the PREVAIL and PROTECT AF trials. J Am Coll Cardiol. 2017; 70(24): 2964-2975, doi: 10.1016/j. jacc.2017.10.021, indexed in Pubmed: 29103847. 
4. de Waha S, Seeburger J, Ender J, et al. Deep sedation versus general anesthesia in percutaneous edge-to-edge mitral valve reconstruction using the MitraClip system. Clin Res Cardiol. 2016; 105(6): 535-543, doi: 10.1007/s00392-015-0951-z, indexed in Pubmed: 26683202.

5. Horn P, Hellhammer K, Minier M, et al. Deep sedation vs. general anesthesia in 232 patients undergoing percutaneous mitral valve repair using the MitraClip system. Catheter Cardiovasc Interv. 2017; 90(7): 1212-1219, doi: 10.1002/ccd.26884, indexed in Pubmed: 28112459.

6. Patzelt J, Ulrich M, Magunia H, et al. Comparison of deep sedation with general anesthesia in patients undergoing percutaneous mitral valve repair. J Am Heart Assoc. 2017; 6(12), doi: 10.1161/JAHA.117.007485, indexed in Pubmed: 29197832.

7. Toppen W, Johansen D, Sareh S, et al. Improved costs and outcomes with conscious sedation vs general anesthesia in TAVR patients: Time to wake up? PLoS One. 2017; 12(4): e0173777, doi: 10.1371/journal.pone.0173777, indexed in Pubmed: 28379981.

8. Hyman MC, Vemulapalli S, Szeto WY, et al. Conscious Sedation Versus General Anesthesia for Transcatheter Aortic Valve Replacement: Insights from the National Cardiovascular Data Registry Society of Thoracic Surgeons/American College of Cardiology Transcatheter Valve Therapy Registry. Circulation. 2017; 136(22): 2132-2140, doi: 10.1161/CIRCULATIONAHA.116.026656, indexed in Pubmed: 28864443.

9. Husser O, Fujita B, Hengstenberg C, et al. Conscious Sedation Versus General Anesthesia in Transcatheter Aortic Valve Replacement: The German Aortic Valve Registry. JACC Cardiovasc Interv. 2018; 11(6): 567-578, doi: 10.1016/j.jcin.2017.12.019, indexed in Pubmed: 29566803.

10. Mosleh W, Mather JF, Amer MR, et al. Propensity matched analysis comparing conscious sedation versus general anesthesia in transcatheter aortic valve implantation. Am J Cardiol. 2019; 124(1): 70-77, doi: 10.1016/j.amjcard.2019.03.042, indexed in Pubmed: 31064667.

11. Fountain RB, Holmes DR, Chandrasekaran K, et al. The PROTECT AF (WATCHMAN Left Atrial Appendage System for Embolic PROTECTion in Patients with Atrial Fibrillation) trial. Am Heart J. 2006; 151(5): 956-961, doi: 10.1016/j.ahj.2006.02.005, indexed in Pubmed: 16644311.

12. Holmes D, Reddy V, Turi Z, et al. Percutaneous closure of the left atrial appendage versus warfarin therapy for prevention of stroke in patients with atrial fibrillation: a randomised non-inferiority trial. The Lancet. 2009; 374(9689): 534-542, doi: 10.1016/s01406736(09)61343-x.

13. Meier B, Blaauw Y, Khattab AA, et al. EHRA/EAPCI expert consensus statement on catheter-based left atrial appendage occlusion. Europace. 2014; 16(10): 1397-1416, doi: 10.1093/europace/ euu174, indexed in Pubmed: 25172844.

14. Mehran R, Rao SV, Bhatt DL, et al. Standardized bleeding definitions for cardiovascular clinical trials: a consensus report from the Bleeding Academic Research Consortium. Circulation. 2011; 123(23): 2736-2747, doi: 10.1161/CIRCULATIONAHA.110.009449, indexed in Pubmed: 21670242.

15. Kappetein AP, Head SJ, Généreux P, et al. Valve Academic Research Consortium-2. Updated standardized endpoint definitions for transcatheter aortic valve implantation: the Valve Academic Research Consortium-2 consensus document. EuroIntervention. 2012; 8(7): 782-795, doi: 10.4244/EIJV8I7A121, indexed in Pubmed: 23022744 .

16. Hicks KA, Mahaffey KW, Mehran R, et al. 2017 Cardiovascular and stroke endpoint definitions for clinical trials. J Am Coll Cardiol. 2018; 71(9): 1021-1034, doi: 10.1016/j.jacc.2017.12.048, indexed in Pubmed: 29495982.

17. Team RC. R: A language and environment for statistical computing. R Foundation for Statistical Computing, Vienna, Austria 2018. https://www r-project org.

18. E. A. S. Daniel E. Ho KI, Gary King. MatchIt: Nonparametric Preprocessing for Parametric Causal Inference. E. A. S. Daniel E. Ho, Kosuke Imai, Gary King. J. Stat. Softw. 2011. http://gking. harvard.edu/matchit.

19. N. G. cobalt: Covariate Balance Tables and Plots. R package version 3.4.1. https://github.com/ngreifer/cobalt. 2018.

20. Zangrillo A, Mazzone P, Oriani A, et al. Noninvasive ventilation during left atrial appendage closure under sedation: Preliminary experience with the Janus Mask. Ann Card Anaesth. 2019; 22(4): 400-406, doi: 10.4103/aca.ACA_145_18, indexed in Pubmed: 31621676.

21. Freeman JV, Varosy P, Price MJ, et al. The NCDR left atrial appendage occlusion registry. J Am Coll Cardiol. 2020; 75(13): 1503-1518, doi: 10.1016/j.jacc.2019.12.040, indexed in Pubmed: 32238316.

22. Reddy VY, Gibson DN, Kar S, et al. Post-Approval U.S. Experience with left atrial appendage closure for stroke prevention in atrial fibrillation. J Am Coll Cardiol. 2017; 69(3): 253-261, doi: 10.1016/j.jacc.2016.10.010, indexed in Pubmed: 27816552.

23. Reddy VY, Holmes D, Doshi SK, et al. Safety of percutaneous left atrial appendage closure: results from the Watchman Left Atrial Appendage System for Embolic Protection in Patients with AF (PROTECT AF) clinical trial and the Continued Access Registry. Circulation. 2011; 123(4): 417-424, doi: 10.1161/CIRCULATIONAHA.110.976449, indexed in Pubmed: 21242484.

24. Boersma LVA, Schmidt B, Betts TR, et al. Implant success and safety of left atrial appendage closure with the WATCHMAN device: peri-procedural outcomes from the EWOLUTION registry. Eur Heart J. 2016; 37(31): 2465-2474, doi: 10.1093/eurheartj/ ehv730, indexed in Pubmed: 26822918.

25. Boersma LV, Ince H, Kische S, et al. Efficacy and safety of left atrial appendage closure with WATCHMAN in patients with or without contraindication to oral anticoagulation: 1-year follow-up outcome data of the EWOLUTION trial. Heart Rhythm. 2017; 14(9): 1302-1308, doi: 10.1016/j.hrthm.2017.05.038, indexed in Pubmed: 28577840. 